\title{
On the problem of calcium depression by phosphorus in flame photometry
}

\author{
(Received 30 March 1958)
}

IN spite of the many investigations on the problem of Ca-depression by the phosphate-ion in flame photometry (of. [1-5]), some essential features of this interference remained to be explained. We have made some additional measurements by spraying $\mathrm{CaCl}_{2}$ solutions, with added phosphoric acid, into an acetylene-air and propane-air flame, at various $\mathrm{Ca}$ and P-concentrations, with the height in the flame as a (significant) parameter. No Cadepression was found when $\mathrm{Ca}$ and $\mathrm{P}$ were sprayed separately, by means of two sprayers in parallel, into the same flame. The outcome of these and other measurements has led us to a detailed hypothesis on the mechanism of this type of anion interference, which satisfactorily explains, among other things, its dependence on Ca concentration, height in the flame, flame temperature and size of the spray-droplets as well as the well-known occurrence of a saturation in the extent of the depression. Conclusions could be drawn as to the reduction or elimination of this depressing effect, which may be useful in practical analysis. In the light of these results, the problem of $\mathrm{Ca}$-depression by added aluminium was also reviewed. A more extensive report will be published elsewhere [6].
Physisch Laboratorium,
C. T. J. Alkemade
Rijksuniversiteit, Utrecht, Holland
M. H. VOORHUIS

\section{References}

[1] Baker G. L. and Johnson L. H., Analyt. Chem. 195426465.

[2] Dippes W. A., Thesis, Princeton University 1954.

[3] Leyton L., Analyst 195479497.

[4] Margoshes M. and Vallee B. L., Analyt. Chem. 195628180.

[5] Tsuchikashi S. and Sekido E., J. Chem. Soc. Japan, 1956 77, 708.

[6] Alkgmade C. T. J. and Voorhuis M. H., Z. anal. Chem. To be published.

\section{Spin-forbidden transitions in the electronic spectrum of acetaldehyde*}

\author{
(Received 7 May 1958)
}

THE electronic spectrum of formaldehyde has recently received considerable experimental $[1,2,3,4,5]$ and theoretical $[6,7,8]$ study. In comparison, acetaldehyde has been neglected, the only attempt at analysis of the near ultraviolet vapour spectrum being due to WALSH [9] using Schou's data [10]. CoHEN and REID [2] observed three weak $\beta$ bands ( $f$ approx. $10^{-8}$ ) at $26,380,25,740$ and $25,220 \mathrm{~cm}^{-1}$ in absorption in formaldehyde which they suggested were ${ }^{1} A_{1} \rightarrow{ }^{3} A_{2}$. More recent work $[4,5]$ has revealed that this system consists of nine double-headed bands of which the four longest wavelength components are probably "hot" bands associated with transitions from a ground state in which the $1746 \mathrm{~cm}^{-1}, \nu_{2}$, vibration is excited. We have attempted to detect the analogous transition in acetaldehyde.

Acetaldehyde (reagent grade), was purified in diffuse light by multiple fractional distillation under nitrogen using a 12 in. column of $\frac{1}{8}$ in. diameter glass helices. Fractionation was to $\pm 0 \cdot 1^{\circ} \mathrm{C}$. The boiling point, refractive index and infrared spectrum [11] all

* The experimental work discussed here was carried out in the Research Laboratories of British Nylon Spinners Ltd., Pontypool, England. 